\title{
La blanchité sous la loupe des écrivains autochtones
}

\section{Corrie Scott, Université d'Ottawa}

La blanchité, en tant qu' « a priori ontologique et épistémologique », est séduisante, selon Aileen Moreton-Robinson, à cause de sa prétention d'un esprit neutre et d'un corps détaché et invisible (81). Par contre, le paraître curieusement objectif de la blanchité oppose un contraste saisissant à la prétendue particularité des connaissances autochtones. Par exemple, trop souvent, la littérature autochtone est considérée comme rapportant des « expériences » plutôt que du savoir. Afin d'équilibrer le poids de ces bagages coloniaux ainsi que de concrétiser la blanchité pour la problématiser, dans cet article qui se veut non exhaustif, je me penche sur plusieurs écrits dans lesquels des écrivains autochtones voient et nomment la blanchité de manière explicite et souvent critique. Commençant par Histoires de Kanatha de Georges Sioui, en passant par Je suis une maudite sauvagesse d'An Antane Kapesh, pour terminer avec des extraits des textes de Virginia Pésémapéo Bordeleau et de Michel Jean, j’analyserai la curiosité, la compassion et la colère qui informent ces réflexions littéraires autochtones sur la blanchité.

Il existe, bien sûr, amplement de descriptions de personnages blancs dans les littératures autochtones qui pourraient être analysées, mais dans ce texte, je me penche sur les mises en fiction non seulement de personnages blancs, mais surtout, de la blanchité en tant que système social injuste. À travers des critiques de l'injustice et celles de l'illusion de la supériorité blanche, les textes à l'étude dans ce chapitre menacent ce que le professeur de littérature américaine Stephen Knadler appelle « les limites territoriales et psychiques de la blanchité » (ix). L'objectif de mon analyse est également de restituer les stratégies variées que les écrivain.e.s emploient pour démasquer la blanchité, ses mensonges et ses ruses, car elle est souvent aussi imperceptible pour les personnes blanches qu'elle est hurlante pour les personnes non blanches. En tant que chercheure blanche, dans cet article, je suis avant tout à l'écoute attentive et active des écrivain.e.s autochtones qui tracent le portrait de la blanchité pour la saisir, mais aussi, pour happer le lecteur au passage. 


\section{Qu'est-ce que la blanchité ?}

Comme l'explique le professeur et auteur cherokee Daniel Heath Justice, « que nous soyons Autochtones ou non-Autochtones, nous avons été formés par la culture dominante à voir la blancheur comme normative et éternelle » (120). En conséquence, toute exploration de la blanchité doit inclure une remise en question des fondements conceptuels sur lesquels cette normativité est construite. Dans un contexte spécifiquement littéraire, par exemple, peu importe l'ethnicité, la langue maternelle ou la provenance des chercheur.e.s, des enseignant.e.s et des étudiant.e.s qui se penchent sur les littératures autochtones, trop souvent, ils et elles sont formés presque exclusivement dans les traditions des études littéraires occidentales avec ses biais méthodologiques et ses préjugés culturels eurocentriques. Dans ce sens, la blanchité constitue un problème-clé pour tout chercheur.e, surtout, mais certainement pas exclusivement s'il ou elle s'intéresse aux littératures autochtones.

La blanchité normalisée et institutionnalisée, mais rarement reconnue par les personnes blanches, a un impact très important sur les personnes autochtones qui la décrivent souvent en détail et depuis longtemps. On pense à Sherman Alexie, romancier, poète et scénariste de la nation Cœur d'Alène/Spokane, qui relate ses expériences en librairie : « Souvent je me retrouve seul dans des librairies où je fais des recherches pour mon travail. Je lève les yeux d'un livre et j'aperçois des visages blancs. C'est effrayant » (79). La poète, auteure et artiste sto:lo Lee Maracle montre elle aussi du doigt son aliénation au sein des institutions blanches : « si l'on veut obtenir le droit d'élaborer des théories, il faut fréquenter leurs institutions pendant plusieurs années, apprendre cette autre langue et désapprendre notre sentiment pour la condition humaine. Étrange » (43). Ces deux exemples évoquent la sensation d'être entouré.e de personnes blanches qui contrôlent la production du savoir et les mécanismes de pouvoir. La blanchité institutionnelle est façonnée par la proximité de certains corps et pas d'autres dans des espaces collectifs ou publics. Quand les personnes blanches dominent largement une institution, elles déterminent les objectifs à poursuivre et les intérêts qui priment. Pour Alexie et Maracle, le caractère institutionnel de la blanchité est une source d'aliénation et d'inégalité.

Un grand nombre de chercheur.e.s et auteur.e.s autochtones ont abordé les inégalités structurelles de la blanchité sous tous ses aspects, tant littéraires que politiques. La grande théoricienne, auteure et professeure crie/métis Emma LaRocque décrit l'objectification 
institutionnelle des personnes autochtones qui sont reconnues pour certaines contributions, mais pas d'autres :

Si nos contributions culturelles et personnelles sont clairement et itérativement reconnues, notre apport au plan théorique, lui, n'est pas accueilli avec la même considération. Persistent ainsi des renvois exagérés à nos « traditions », ou à notre « expérience» coloniale ou personnelle, ces dernières faisant l'objet de généralisations ou de retraductions supplémentaires. Ce qu'il manque à ces travaux, selon moi, ce sont nos recherches, nos appareils critiques, nos questionnements et nos idées. (199)

Si LaRocque décrit le manque de reconnaissance des apports théoriques autochtones, Alexie, quant à lui, témoigne d'une des conséquences de ce déséquilibre structurel : «Chaque fois que je m'aventure dans une librairie, je tombe sur un autre livre à propos des Indiens. Il existe des centaines de livres sur les Indiens publiés chaque année, mais si peu sont écrits par des Indiens » (77). Dans la même veine, Maracle insiste sur l'inégalité des critères d'évaluation : « Notre façon d'utiliser les mots et la langue n'est pas évaluée selon des critères établis par la poésie et les histoires que nous avons inventées. Il s'agit de modes d'évaluation et de critères établis par d'autres » (135). Tour à tour, chacun.e de ces théoricien.ne.s aborde ce problème sous des angles variés. Mais de façon ferme et constante, avec énormément de persévérance, les savants autochtones revendiquent haut et fort le respect de l'idée que l'analyse des littératures autochtones nécessite des outils théoriques autochtones.

Bref, les appareils critiques qui guident étudiant.e.s et chercheur.e.s dans leurs lectures de littératures autochtones ont été principalement développés par des érudits blancs qui se positionnent en tant que spécialistes ou experts. Dans ce sens, la blanchité n'est pas simplement réductible à des personnes blanches; il s'agit d'un système hégémonique et suprémaciste qui confère une dominance aux Blanc.he.s. Pour les littéraires, l'importance est on ne peut plus claire. L'inégalité dont on parle n'est pas une simple question de manque de représentation, même si, cette dimension de la production littéraire au Québec doit «cesser de porter le visage l'innocence », comme le disent Carole David et Martine Delvaux (2017). Pour équilibrer un tant soit peu les inégalités identifiées par les savants autochtones cités plus haut, ce qu'il faut, pour utiliser les termes de la théoricienne anglophone Sara Ahmed, ce sont des interventions dans «la manière dont le racisme répartit les ressources et les capacités de manière inégale. Ces répartitions inégales affectent également le 'business' de la parole et qui peut dire quoi, à propos de qui et où » (« Declarations of Whiteness », n.p. ; ma traduction $)^{1}$. 
Un petit détour vers les théories de la blanchité développées par les féministes racisées américaines s'avère également utile. Hazel Carby, pionnière dans le domaine du féminisme noir et des études afro-américaines, appelle l'état normatif et évaluatif de la blanchité, « le point blanc dans l'espace à partir duquel nous avons tendance à identifier la différence » $(193)^{2}$. Audre Lorde, féministe afro-américaine célèbre, parle d'une «norme mythique» $(116)^{3}$. Et pour Ahmed, féministe queer et racisée, «la blanchité pourrait être décrite comme une histoire en cours et inachevée qui oriente les corps dans des directions spécifiques» ("Phenomenology of Whiteness », 149 ; ma traduction) ${ }^{4}$. Un point dans l'espace, une norme illusoire, ou bien une orientation - toutes ces façons de décrire la blanchité la présentent en tant que pratiques évaluatives supposément objectives derrière lesquelles se cachent les spécificités et les privilèges des personnes blanches. La blanchité est alors le plus souvent un centre non marqué, ce qui permet aux personnes blanches d'éviter le regard perçant de l'autre, mais aussi, de protéger les avantages acquis sous ce régime. bell hooks, féministe afro-américaine de grand renom, note que les Blanc.he.s deviennent parfois très défensifs lorsque l'attention est attirée sur leur blanchité, lorsqu'ils et elles sont vus par des personnes non blanches comme des Blanc.he.s :

Souvent, leur rage éclate parce qu'ils croient que toutes les manières de voir qui mettent en évidence la différence renversent la croyance libérale en une subjectivité universelle (nous ne sommes que des personnes) qui, selon eux, fera disparaître le racisme. Ils ont un investissement émotionnel profond dans le mythe de la « similitude », même si leurs actions reflètent la primauté de la blanchité en tant que signe informant qui ils sont et comment ils pensent. $(167 \text {; ma traduction })^{5}$

Malgré la prétendue imperceptibilité de la blanchité, pour survivre à la violence de la suprématie blanche, les personnes racisées et autochtones ont souvent développé une connaissance remarquable de toute une variété de comportements, de systèmes, d'histoires, de pratiques culturelles blancs. Si ce sont des érudits blancs qui se positionnent depuis longtemps comme les principaux chercheur.e.s sur la vie, les valeurs et les capacités des peuples autochtones, cette foisci, en inversant cette relation sujet-objet, j'examinerai la blanchité sous la loupe des savants autochtones.

\section{L'axe de la différence retracé}

Je commence avec une analyse de plusieurs des multiples essais et discours du grand historien et auteur wendat Georges Sioui rassemblés dans Histoires de Kanatha : vues et contées dans lequel Sioui nous livre une perspective wendate de l'histoire qui s'avère large et englobante. 
Je me permets l'inclusion des essais de Sioui, historien, dans cet article qui porte avant tout sur la littérature autochtone, parce que ses écrits sont assez riches, philosophiques et innovateurs pour y voir une forme littéraire. Mais aussi, ses analyses des pratiques européennes historiques sont révélatrices et, dans le présent article, servent également à contextualiser et à historiciser la blanchité en Amérique du Nord. Pour commencer, il est à noter que Sioui fait bien sûr une distinction entre les différents colonisateurs du continent, sans les homogénéiser, mais aussi, sans que ces distinctions priment :

L'Amérique anglo-saxonne et l'Amérique francophone, toutes deux nordiques, sont assez différentes [...] Leur histoire s'est distinguée par une certaine distanciation idéologique qui a permis (et ce, beaucoup plus dans le cas de l'Amérique française) une certaine idéalisation des premiers peuples, même s'il est certain que cette admiration ne fut parfois rien de plus qu'un outil rhétorique inventé et utilisé pour critiquer et attaquer « les plus respectables » institutions des mères-patries. (216217)

Si l'Amérique anglo-saxonne et francophone sont différentes l'une de l'autre, ainsi que des autres cultures colonisatrices, une chose qu'elles ont en commun, c'est leur persécution des peuples autochtones. Sioui reconnaît ce qu'il appelle la « rencontre philosophique » unique et privilégiée entre les penseurs français et les Autochtones dans la colonie française laurentienne, mais il souligne également que l'Amérique française (c'est-à-dire la Nouvelle-France, ancêtre du Québec) « eut un effet physique tout aussi désastreux» que les autres forces colonisatrices sur les populations autochtones (217). C'est ainsi que se met en place une critique de l'histoire coloniale en Amérique du Nord qui déplace son centre de gravité d'une dispute entre Anglo-saxons et francophones avec les Autochtones en marge, à la résistance des Premières nations face aux violences des multiples forces colonisatrices. Au-delà des différences entre les colonisateurs, l'axe de la pensée de Sioui divise avant tout les nations autochtones de leurs colonisateurs.

Si certains auteur.e.s blanc.he.s favorisent un récit de rapprochement entre l'Amérique française et les Premières nations pour se distinguer des Anglophones et des «Français de France $»^{6}$, beaucoup d'auteur.e.s franco-autochtones ont tendance à brosser le tableau d'une blanchité commune malgré certaines nuances. Par exemple, Sioui explique la différence fondamentale entre les nouveaux arrivants européens et les Premières nations par la discordance entre ce qu'il appelle la vision du monde «linéaire » européenne et la vision panamérindienne « circulaire » : 
Le Cercle est ce qui distingue essentiellement la pensée des Amérindiens et des autres peuples naturels par opposition à la pensée linéaire qui est venue d'Europe. Le Cercle veut simplement dire que l'homme qui possède cette façon de voir est capable de reconnaître la parenté qui existe entre toutes les formes de vie, non seulement entre les êtres humains. Il est capable de voir que sa propre existence dépend du bien-être des autres êtres. Les penseurs linéaires, pour leur part, ont perdu cette capacité de concevoir la relation et la parenté qui nous unissent à tous les autres êtres. (75)

Durant sa longue et belle carrière d'historien, Sioui décrit et analyse l'arrivée subite et violente d'envahisseurs et de leurs cosmovisions linéaires et patriarcales parmi une civilisation WendatIroquois circulaire et matricentriste. Les penseurs linéaires, venus de l'Europe, ont perdu de vue la filiation entre toutes les formes de vie, la notion de complémentarité et d'interdépendance. Source de souffrance et d'agression, la pensée linéaire représente une différence d'approche qui recouvre, autrement dit, une dichotomie profonde qui oppose Européen et Autochtone, Blanc et Panamérindien. Sioui ne présente pas cette différence comme étant essentielle, c'est-à-dire, innée ou intrinsèque, mais elle est toutefois profonde « au plan sociobiologique » (45) et relative aux comportements sociaux liés au rythme de la terre sacrée, « terre de vie pour tous » (47).

S'il n'existe pas d'expérience fixe qui différencie automatiquement une personne autochtone en fonction de sa peau, les auteur.e.s autochtones étudié.e.s dans cet article reconnaissent certaines expériences partagées qui découlent du fait que, peu importe de quelle nation il ou elle vient, ils ont tous été affligés par les institutions blanches et, par conséquent, la résistance à la blanchité est commune à l'ensemble. On pense à Je suis une maudite sauvagesse de l'écrivaine innue An Antane Kapesh, essai d'importance incontournable et même visionnaire pour sa volonté politique et poétique. Dans ce texte publié en 1976 en innu et en français, Antane Kapesh déclare audacieusement, dès la première phrase du préambule, « Dans mon livre, il n’y a pas de parole de Blancs » (13). Cette énonciation est cruciale pour le lancement de son livre qui nomme explicitement la blanchité pour rejeter sa suprématie et les récits qui la soutiennent. Précurseure injustement méconnue des études critiques de la blanchité, Antane Kapesh dénonce ouvertement la domination blanche pour la dénaturaliser, vingt ans avant que les spécialistes blancs de la blanchité américaine tentent de faire la même chose.

Pour déloger la blanchité de sa position de pouvoir et pour l'ébranler de son autorité, Antane Kapesh met en relief les mécanismes et les institutions qui permettent aux personnes blanches de maintenir une position dominante cachée derrière la prétendue neutralité. Chaque section est 
consacrée à une institution pour la problématiser : l'éducation, les dysfonctionnements judiciaires et policiers, l'extraction des ressources naturelles, les médias, le logement, pour n'en nommer que quelques-unes. Selon Isabella Huberman, chercheure blanche qui étudie les littératures autochtones francophones, «toutes les structures et organisations qui lui apparaissent injustes et aliénantes pour les Premières Nations sont condamnées par Antane Kapesh » (113). Par exemple,

En 1969, j'ai vu deux policiers de la Ville rentrer mon fils au poste de police. J'avais déjà entendu dire que les policiers - ceux de la police municipale et ceux de la Gendarmerie royale - battaient les Indiens, jeunes et adultes, quand ils les arrêtaient. Après que les deux policiers municipaux ont rentré mon fils au poste de police, j'y suis allée avec une de mes filles. En entrant, nous voyons les deux policiers battre mon fils avant de le mettre en cellule. Ils le frappent tous deux dans le ventre et partout sur le corps. Ma fille et moi nous mettons alors à pousser des cris. En nous entendant crier et en nous voyant, les policiers cessent de battre mon fils et vont correctement l'enfermer dans une cellule. (Antane Kapesh 81)

Ce passage fait preuve d'une longue histoire de violence blanche envers les personnes autochtones en Amérique du Nord qui continue aujourd'hui. Malgré le fait que ce passage date de 1969, on sait que les personnes autochtones sont encore victimes de violence et de profilage racial policier aujourd'hui. Peu importe de quelle force policière il s'agit, les policiers municipaux ou bien ceux de la Gendarmerie royale, on y retrouve une même culture de racisme, de domination violente et de dissimulation. Antane Kapesh discerne les forces policières locales des forces policières fédérales, mais leur violence envers les Innu.e.s est un tort partagé.

Pour signaler l'ampleur du problème, Antane Kapesh note également l'absence de protection institutionnelle pour les personnes innues ainsi que des alliances entre Blancs :

Quand les policiers nous malmènent de cette façon, ils n'ont personne à craindre : parmi les Blancs qui sont à notre service, il n'y a vraiment personne pour nous protéger et nous défendre, nous les Indiens. Les fonctionnaires qui travaillent pour nous ne pensent qu'à leur salaire et qu'à travailler secrètement pour le Blanc. Il nous est difficile de savoir - et peut-être ne le saurons-nous pas avant notre mort-le nombre de Blancs de Québec et d'Ottawa qui sont venus travailler pour nous. Et nous ignorons par quel discours on les a enrôlés, là-bas d'où ils viennent, dans les services aux Indiens. Ce qui nous étonne c'est que, depuis des années que les policiers nous maltraitent, jamais, parmi les Blancs qui travaillent pour nous, nous n'en avons trouvé un seul pour prendre notre défense. Je pense que tous les Blancs qui ont travaillé pour nous, les Indiens, n'étaient bons qu'à nous pousser à notre insu pour que, bon gré mal gré, nous nous soumettions entièrement au régime des Blancs. $(78-80)$

Antane Kapesh dénonce ce complot ourdi par les Blancs d'Ottawa et ceux de Québec, motivés par leur salaire à entretenir le régime des Blancs au détriment des Autochtones. En identifiant ce 
mécanisme économique qui sert à motiver les Blancs, Antane Kapesh expose succinctement un régime de suprématie blanche avec deux maîtres, ceux d'Ottawa et ceux de Québec. Ces deux piliers soutiennent la suprématie blanche, en partie grâce aux systèmes judiciaires, y compris le système de justice pénale canadien et québécois : «Durant les années où les policiers de la Municipalité et ceux de la Gendarmerie royale ont travaillé pour nous », Antane Kapesh se demande, «pour quelle raison n'y a-t-il qu'avec nous, les Indiens, que le Blanc remplit constamment ses prisons? Chaque fois qu'on nous a passés en cour en même temps que des Blancs, il y avait toujours plus d'Indiens que de Blancs devant le tribunal blanc» (81). Malheureusement, les statistiques corroborent toujours les inégalités que décrit l'écrivaine. En fait, les iniquités de longue date dans le système de justice pénale se sont s'aggravées depuis la publication de Je suis une maudite sauvagesse en 1976 même si la proportion d'Autochtones dans les pénitenciers a augmenté (Jourdan 2020). Mais notons qu'Antane Kapesh précise encore une fois que ce sont les policiers «de la Municipalité et ceux de la Gendarmerie royale » qui surchargent les prisions d'« Indiens ». Dans Je suis une maudite sauvagesse, la blanchité est localisée au Québec et à Ottawa.

Antane Kapesh dénonce également le système d'éducation qui engendre des inégalités sociales pour promouvoir le colonialisme. Dans son rapport final en 2015, la Commission de vérité et réconciliation du Canada (CVRC) a sensibilisé le public canadien sur les pensionnats indiens avec ses témoignages d'enfants déracinés, de parents oubliés et de pensionnats surpeuplés, sousfinancés et souvent insalubres. Mais bien avant, dans Je suis une maudite sauvagesse, Antane Kapesh déplorait déjà le fait que les enfants innus étaient traités comme des pions dans un jeu politique entre deux gouvernements :

Quand, au début, on a pris nos enfants pour leur donner une éducation de Blanc, il est vrai qu'ils relevaient exclusivement du gouvernement fédéral. Quelques années plus tard, nous ne nous sommes même pas rendu compte qu'on les transférait à la commission scolaire. Quand le Blanc a songé à ce transfert, il ne nous a pas consultés, nous les Indiens. Une fois nos enfants transférés à l'école provinciale, le Blanc a démoli le pensionnat qu'il avait auparavant construit pour nous à Sept-l̂les. (78)

Bref, Antane Kapesh fait une distinction entre le gouvernement fédéral et la commission scolaire provinciale, mais il existe néanmoins des similitudes troublantes. Aucun des deux n'a consulté les Innus avant de prendre des décisions à l'égard de leur système éducatif. Et dans les pensionnats, 
peu importe qui les dirigeait, les élèves innus n'avaient pas le droit de parler leur propre langue et la politique d'assimilation régnait :

Quand le Blanc a pris nos enfants pour les éduquer à sa manière, il ne nous a pas dit ceci : «Vous, les Indiens, êtes-vous d'accord que je donne à vos enfants une éducation de Blanc dans le but unique de détruire leur culture et leur langue? [...]» Le Blanc n'a pas parlé de cela à l'Indien. Ce qu'il ne lui a pas dit, c'est qu'il voulait tuer notre culture à notre insu, il voulait tuer notre langue à notre insu et il nous volait notre territoire. (17-18)

La chercheure et professeure blanche Isabelle St-Amand a raison de souligner comment Antane Kapesh fait connaître «les intentions malhonnêtes » et «cachées » du Blanc en décrivant ses actions telles que perçues et éprouvées par sa communauté (39). Effectivement, « Kapesh cherche à dévoiler la duplicité, les injustices et la répression qui ont soutenu le processus de colonisation » (39). La duplicité, la dépossession et la destruction des langues et cultures autochtones était, selon Antane Kapesh, le propre du Blanc, peu importe qui gouvernait l'école.

De plus, en déclarant sa « souveraineté individuelle» (116), pour utiliser l'expression de Huberman, Antane Kapesh condamne la violence et la duplicité des deux faces de la même médaille de la blanchité canadienne. Peu importe de quelle force policière il s'agit, on y retrouve une même culture de domination. Peu importe quel gouvernement se chargeait de l'éducation des enfants autochtones, leur langue était interdite. Quand Sioui identifie la pensée circulaire comme trait partagé par les diverses Premières Nations, c'est pour se distinguer de la pensée linéaire qui est venue d'Europe avec les colonisateurs sous plusieurs drapeaux. Bref, Antane Kapesh et Sioui ne confondent aucunement les différents peuples colonisateurs et la distinction qu'ils opèrent est claire. Mais quand les écrits d'un historien wendat et d'une écrivaine innue se trouvent au cœur de notre réflexion, un des effets est le déplacement de ce « point blanc dans l'espace à partir duquel nous avons tendance à identifier la différence »(Carby 193). Recadrés avec ces perspectives autochtones au centre, les similitudes entre les peuples colonisateurs sont troublantes : domination, violences, duplicités et injustices. Deux piliers émergent pour soutenir la charpente de la blanchité dans ce qu'on appelle actuellement le Canada.

\section{L'Europe, lieu de conflits, maladies et malheurs}

Le cadre conceptuel ainsi déplacé nous permet également de troubler l'autoportrait flatteur dressé par le Blanc qui camoufle habilement sa violence. Par exemple, l'autodescription de l'arrivée des Européens en Amérique du Nord que l'on retrouve dans les manuels scolaires est 
généralement affublée d'adjectifs comme aventureux, courageux, civilisateur là où les penseur.e.s autochtones parlent de conquérants, d'agresseurs et de traitres, et ce, en parlant des colonisateurs francophones et anglophones. Mais quand on situe les «aventures» européennes dans une perspective wendate, on aboutit à l'image remarquable et récurrente des Blancs pas en tant qu'aventuriers, mais en tant que réfugiés fuyant une culture malsaine. "'Mens sana in corpore sano', disons-nous tous » (45), écrit Sioui. Il explique :

De grandes parties de l'Europe, au moment où ce continent arriva accidentellement en Amérique, n'étaient qu'un grand foyer d'épidémies, tellement la Ligne avait intégralement remplacé le Cercle. On pourrait même dire que l'Europe, chroniquement et mortellement malade, a frénétiquement cherché un remède et son salut à la fin du XVe siècle. (46)

Plutôt qu'aventuriers, les Européens deviennent, dans cette perspective, des expatriés malades fuyant « un vieux monde » parce qu'il « ne promettait que la mort » (47), ce qui explique, toujours selon Sioui, leur violence :

Et l'insensibilité morbide des découvreurs et de leurs suites vis-à-vis de l'existence, de la disparition et du droit des Peuples originaires, ainsi que vis-à-vis de toute forme de vie en général, ne doit apparaître que comme le corollaire d'un état physique également morbide d'une grande quantité d'Européens de ces siècles. Car qu'indiquent l'agressivité insensible, le mépris de la vie, la soif insatiable et aveugle de possessions matérielles, le goût de l'anéantissement et toutes les autres pathologies mentales antisociales, sinon une biologie souffrante, exaspérée ? (45)

Dans cette image sans doute peu enviable, on note malgré tout que l'agressivité, le mépris et les autres pathologies que Sioui attribue aux « découvreurs » ne représentent aucunement une essence ou une caractéristique naturelle des Blancs, même s'il s'agit pour Sioui de la marque principale de la civilisation eurogène en Amérique (45). Ces arrivants européens sont victimes d'une « biologie souffrante » causée par la pensée linéaire et la destruction du monde naturel, source de leur souffrance et de leur agressivité.

Sioui est loin d'être le seul écrivain autochtone à brosser un tel portrait de colonisateurs blancs malsains. Professeure de la nation Métis, Jo-Ann Episkenew conçoit la littérature autochtone comme remède contre ce qu'elle appelle « la contagion coloniale » (170). Elle précise :

Le colonialisme est malade ; sous ses auspices et soutenus par sa mythologie, les colonisateurs ont infligé d'odieuses blessures aux populations autochtones qu'ils cherchaient à civiliser. Même si les Autochtones comprennent le besoin qu'ils ont de se remettre du traumatisme de la colonisation, la plupart des colons nient que leur société est construite sur des bases malsaines et qu'elle a donc besoin d'un traitement. (182) 
Souvent, notre conception du colonialisme repose surtout sur les dommages infligés aux personnes autochtones, mais la mise au point d'Episkenew permet de cibler la source du problème et pas simplement ses effets. Pour d'autres écrivains autochtones, l'Europe est également un monde de conflit et de malheurs. On pense au roman L'amant du lac de Virginia Pésémapéo Bordeleau, romancière métisse crie prolifique, qui s'ouvre avec le viol brutal du personnage principal Waseshkun, jeune femme algonquine, par une Robe noire (un missionnaire jésuite) quand il était en visite au village de Pointe-aux-Pins (23). Des années plus tard et vers la fin du roman, un autre personnage, Gabriel, qui est d'origine française et abénaquise (et l'amant de Wabougouni, petite fille de Waseshkun), part pour participer à « une guerre en ces pays lointains, au-delà des mers d'où son peuple était originaire, le peuple à peau blanche » (59). Mais il devient lui aussi victime d'un viol par un homme parisien, cette fois-ci en sol français. Le thème du viol semble avoir une fonction structurante dans le roman qui illustre la continuité de la violence coloniale à travers des époques distinctes. Ces abus sexuels contrastent fortement avec la réciprocité entre Autochtones et le monde naturel du lac Abitibi, comme le souligne la chercheure blanche Hilary Walton dans son analyse du roman. Pour Walton, « le viol représente l'inverse d'une sexualité saine puisque la violence qui le caractérise va à l'encontre de tous les principes qui régissent la sexualité autochtone » (22). Dans L'amant du lac, le viol paraît alors être le symptôme d'un mal chronique qui ronge la culture européenne et ses descendants des deux côtés de l'Atlantique.

Le lendemain du viol de Gabriel, il se réveille, saignant et tout seul. « Alors la colère monta » (127). «Il marcha dans toutes les pièces, nu, son poignard serré contre lui, de son pas silencieux lorsqu'il devait débusquer le nazi » (128). Or, Gabriel ne fait pas face à l'ennemi nazi dans le « décor luxueux » (127) de cette chambre parisienne. Il se met plutôt à l'attaque des objets de luxe qui l'entourent :

Il éventra les fauteuils en cuir, le matelas du lit, les oreillers de plumes, découpa les rideaux de brocart et de dentelle, les draps de soie, les tapis d'Orient, les dizaines de beaux habits et les chemises de la garde-robe, coupa les cravates avec le même geste que l'éclaireur tranchant le cou des soldats ennemis pris par surprise. Il égratigna les meubles en bois précieux, y planta le couteau, cassa les bibelots de porcelaine chinoise, les vitraux sur les portes, lança sur les murs les bouteilles millésimées, renversa le buffet avec la vaisselle de Limoges. (128)

Les tissus et les tapis, les bibelots et les bouteilles de grande valeur... De cet inventaire très détaillé des objets détruits par Gabriel découle un renforcement référentiel difficilement négligeable. Certains objets teintés d'exotisme orientaliste, comme la porcelaine chinoise et les tapis d'Orient, 
font peut-être allusion au colonialisme français. D'autres détails, comme le cuir, la dentelle, la soie ainsi que le bois précieux dans lequel il plante son couteau, servent à concrétiser une forme de luxe ostentatoire français dans toute sa particularité. Pésémapéo Bordeleau situe le viol de Gabriel dans cet ancrage référentiel sans doute pour créer une ombre de vraisemblance, mais aussi, symboliquement, pour exposer le côté sinistre de l'opulence culturelle française.

Toutefois, malgré ce portrait sombre de la culture du colonisateur français, Pésémapéo Bordeleau nourrit une lueur d'espoir. Vers la fin de la crise de colère de Gabriel, « il leva les yeux vers les tableaux de maîtres, il hésita, le bras en l'air, la lame prête à crever la toile » (128). Mais « après un long moment, sa main retomba. Il glissa le poignard à sa ceinture, prit sa veste et s'en alla sans se retourner. Il n'avait pas touché à la bibliothèque ni aux livres » (128). Ce geste symbolique me semble très généreux dans le contexte du colonialisme. Si la décadence de la culture française est ciblée par la colère de Gabriel, les lettres et les arts échappent de peu à sa juste vengeance. D'ailleurs, il faut sans doute se rappeler que Gabriel est lui-même d'origine française et abénaquise, l'identité métisse étant un thème qui figure souvent dans la fiction de Pésémapéo Bordeleau. Existant sur la frontière de plusieurs cultures, le personnage de Gabriel s'est engagé dans une guerre lointaine, « d'où son peuple était originaire, le peuple à peau blanche » (59). Mais il revient de « l'enfer des hommes, pire que celui du diable ou de Dieu » pour rejeter la doctrine et l'autorité de cette guerre : «Qui a dit que l'honneur était au bout du fusil ? Mensonges, leurres, duperies!» (129).

Quand une femme de chambre d'hôtel lui apporte du bouillon pour soigner ce qui reste de « la zone dévastée de son âme » (129-130), Gabriel décide de retourner vers le lac Abitibi et sa bien-aimée algonquine, Wabougouni. «D’un retour vers la terre, après la défaite des rêves de gloire, croûtes mortes qui s'envolaient au vent de la certitude d'être sur la bonne voie. Le métis sentait ses blessures se cicatriser au fur et à mesure que la distance entre lui et sa bien-aimée se réduisait » (135). Dans L'amant du lac, la blanchité n'est alors pas réductible à des personnages blancs «méchants». Gabriel, un Blanc qui n'est pas blanc, doit lutter dans sa relation à la blanchité. À la fin du roman, il renouvelle son allégeance à la culture algonquine pour goûter aux plaisirs de la vie et de l'amour au mitan de sa vie. Pésémapéo Bordeleau joue sur le contraste entre un univers algonquin sain et l'univers malsain du «peuple à peau blanche» (59). Gabriel, Abénaquis par sa mère et Blanc par son père, finit par tourner le dos à la brutalité patriarcale du monde des Blancs pour s'orienter vers la terre mère algonquine et l'amour de Wabougouni. 
Pourtant, s'orienter vers la blanchité, ou s'en détourner, n'est pas réductible à un simple principe de libre choix. Pour un exemple des contraintes imposées par le régime de la blanchité, passons au beau roman Elle et Nous de l'écrivain innu et blanc Michel Jean. En discutant du roman lors d'un entretien, l'auteur avoue, « je suis un Innu », avant d'ajouter « avec un peu de timidité », « mais pendant longtemps, c'est quelque chose dont je ne parlais pas » (Fauteux 2019). Pour comprendre pourquoi, il faut lire le roman qui alterne entre deux narrateurs : elle et lui. Elle est inspirée de la grand-mère de l'auteur. Le personnage Shashuan Pileshish, ou Hirondelle en innu, est « née entre les arbres, sur les berges gelées du lac Péribonka » (225) sur la rive nord du Lac Saint-Jean. Lui, l'autre voix narrative, est le petit-fils de Shashuan Pileshish qui s'appelle Michel. À l'âge adulte, en visite chez sa grand-mère, Michel se demande pourquoi elle a rétorqué sèchement, « Je ne suis pas indienne, moi !» (103) quand il la présentait à un ami. Avec Michel, on se demande alors, qu'est-ce qui a poussé Shashuan Pileshish à désavouer son identité innue?

Un retour dans le temps est nécessaire pour répondre à cette question. À dix-sept ans, Shashuan Pileshish tombe en amour avec François-Xavier Gagnon, « élevé comme un Canadien » (223) et Blanc en ce qui concerne « le statut et la culture » (206), mais aussi, fils illégitime d'un Innu. Quand elle a vu son amoureux pour la première fois, le cœur serré, les jambes tremblantes et la tête tournante, le moment l'a « fait basculer dans un autre monde. Le sien, à lui » (205). La Loi sur les Indiens faisait en sorte qu'elle devait quitter sa communauté et perdre son statut d'Autochtone après les noces. Cette rupture douloureuse correspond à un changement de nom (Shashuan Pileshish, ou Hirondelle, devient Jeannette Gagnon) et, aussi, à un déménagement en ville, à Alma.

Malgré leur grand amour et certains soutiens familiaux, en ville, les enfants ont vécu un racisme douloureux. Lors du défilé de la Saint-Jean-Baptiste, par exemple, une de ses filles devait jouer « le rôle d'une Indienne sur un char allégorique représentant les saints martyrs canadiens. Elle avait reçu les insultes comme des coups de couteau dans le ventre. Elle était rentrée ce jourlà les yeux rougis, le cœur gros comme le Pekuakami »(226-227). Désespérée, Shashuan Pileshish avoue :

J'en suis venue peu à peu à regretter des origines qui autrefois étaient ma fierté, mais qui maintenant faisaient souffrir mes petits. J'ai fait des efforts pour les dissimuler le plus possible. Je me suis mise à porter des vêtements comme les autres, à me coiffer comme mes voisines. Outre ma peau plus foncée, et dont on pouvait attribuer la couleur aux longues journées passées au soleil, j'avais l'air d'une parfaite Canadienne. Enfin presque. Shashuan Pileshish Siméon est peu à peu devenue 
Jeannette Gagnon. Souvent, si l'on me demandait si j'étais indienne, je le niais tout simplement. Je mentais sur qui j'étais. Par amour. (227-228)

Pour protéger ses enfants des abus racistes, Shashuan Pileshish dissimule autant que possible ses origines innues pour devenir Jeannette Gagnon, femme blanche. Dans le jargon du racisme américain, elle « passait ». Le "passing » est un phénomène bien connu des Noir.e.s aux ÉtatsUnis et est une caractéristique de la subordination raciale dans toutes les sociétés structurées par la suprématie blanche. Dans le très connu « Whiteness as Property », la professeure des droits civiques et théoricienne noire américaine Cheryl I. Harris médite longuement sur le «passing » de sa propre grand-mère et sur l'effacement de son identité noire. Elle explique :

La décision de passer n'était pas un choix, si par ce mot on entend le caractère volontaire ou le manque de contrainte. Le fait de la subordination raciale était coercitif et limitait la liberté de se définir. L'autodétermination identitaire n'est pas un droit pour tous, mais un privilège accordé sur la base de la race. La protection de la blanchité juridique a eu pour effet de dévaluer ceux qui n'étaient pas blancs en les contraignant à nier leur identité pour survivre. (1743; ma traduction) ${ }^{7}$

Pour Harris, le passing est «la manœuvre assimilationniste ultime ${ }^{8}$ (1765) et nécessite la complicité et le risque de l'auto-annihilation. Devenir blanc.he donne sans doute accès à un ensemble d'acquis, d'avantages, de gains et de contrôle de sa vie plutôt que d'être l'objet d'insultes et d'insinuations racistes. Alors, on comprend pourquoi Shashuan Pileshish se rend invisible pour se mettre à l'abri de la blanchité et pour étendre cette protection à ses enfants. Devenir blanche était une façon pour elle de survivre à la suprématie blanche : "Je pouvais m'effacer sans disparaître. Je sais bien que ma conduite a pu paraître étrange parfois. J'ai créé une autre moi, mieux adaptée à ma nouvelle vie. Une façon de survivre » (230). L'identité, dans ce cas, est un jeu truqué soumis aux contraintes considérables de la blanchité. Si dans L'amant du lac, Gabriel arrive à se diriger vers la terre mère pour rejeter la blanchité de ses ancêtres, dans Elle et Nous, ce genre d'autodétermination identitaire n'est guère disponible pour Shashuan Pileshish.

\section{Conclusion}

Plutôt que de percevoir un pays divisé entre «deux solitudes» qui place les deux populations blanches au centre de l'histoire, Sioui, Antane Kapesh, Pésémapéo Bordeleau, Jean et tant d'autres auteur.e.s autochtones construisent des univers dans lesquels le sens gravite autour d'un noyau autochtone. Il n'y a rien d'essentiel dans cet encadrement qui fonctionne à plein régime pour dévoiler un centre blanc non marqué, pour le dénaturaliser, mais aussi, pour voir autrement. 
Que nous soyons Autochtones ou non-Autochtones, la primauté de la blanchité, en tant que signe informant qui nous sommes et comment nous pensons, comporte des risques inégaux pour tout le monde. Mais il est de notre intérêt à tous et à toutes de mettre les savoirs autochtones au premier plan, d'y prêter l'oreille pour mettre « au défi les prémisses et les pratiques épistémologiques et canoniques à l'Occidentale » (LaRocque 196), car en fin de compte, la situation est grave et urgente : «De nos jours, les idées à l'origine du mythe de la supériorité blanche présentent un danger à la survie de l'espèce humaine » (Episkenew 175).

\section{Bibliographie}

Ahmed, Sara. "Declarations of Whiteness : The Non-Performativity of Anti-Racism ». Borderlands 3.2 (2004). http://www.borderlands.net.au/vol3no2_2004/ahmed_declarations.htm

---. « A Phenomenology of Whiteness ». Feminist Theory 8.2 (2007). 149-168.

Alexie, Sherman. « Autobiographie non autorisée de moi-même ». Jeannotte, Lamy et St-Amand. 69-82.

Antane Kapesh, An. Eukuan nin matshimanitu innu-iskueu / Je suis une maudite sauvagesse. Montréal : Leméac, 1976.

Carby, Hazel. « The Multicultural Wars ». Black Popular Culture. Dir. Gina Dent. Seattle: Bay P, 1992. 187-199.

David, Carole et Martine Delvaux. « Blanches ». À Babord! Revue sociale et politique 67 (20162017). https://www.ababord.org/Blanches

Episkenew, Jo-Ann. « Mythe, politique et santé ». Jeannotte, Lamy et St-Amand. 169-192.

Fauteux, Nicolas. « Michel Jean : préserver la mémoire du peuple innu ». Agence QMI, TVA Nouvelles. 6 décembre 2019. https://www.tvanouvelles.ca/2019/12/06/michel-jeanpreserver-la-memoire-du-peuple-innu

Harris, Cheryl I. « Whiteness as Property ». Harvard Law Review 106.8 (1993). 1707-1791.

Heath Justice, Daniel. « Voir (et lire) rouge : les Indiens hors-la-loi dans la tour d'ivoire ». Nous sommes des histoires: Réflexions sur la littérature autochtone. Montréal : Mémoire d'encrier, 2019. 105-130.

hooks, bell. «Representations of Whiteness in the Black Imagination ». Black Looks : Race and Representation. Boston: South End P, 1992. 165-78. 
Huberman, Isabella. «"Si ce n'est pas moi" : écrire à la jonction du soi et de la communauté chez An Antane Kapesh et Natasha Kanapé Fontaine ». Studies in Canadian Literature / Études en littérature canadienne 43.1 (2018). 108-127.

Jean, Michel. Elle et nous. Montréal : Libre Expression, 2012.

Jeannotte, Marie-Hélène, Jonathan Lamy et Isabelle St-Amand, dir. Nous sommes des histoires : réflexions sur la littérature autochtone. Montréal : Mémoire d'encrier, 2019.

Jourdan, Thibault. «Les Autochtones représentent $30 \%$ des prisonniers fédéraux, un 'sommet historique’ ». Radio Canada. 21 janvier 2020. https://ici.radio-canada.ca/nouvelle/1484239/autochtones-prison-delinquance-prairieprison-racisme

Knadler, Stephen P. The Fugitive Race : Minority Writers Resisting Whiteness. UP of Mississippi, 2002.

LaRocque, Emma. « Décoloniser les postcoloniaux ». Jeannotte, Lamy et St-Amand. 193-206.

Lorde, Audre. Sister Outsider : Essays and Speeches. Trumansburg, NY: The Crossing P, 1984.

Mailhot, Amélie-Anne. "La perspective de l'habitation politique dans Je suis une maudite sauvagesse / Eukuan nin matshimanitu innu-iskueu d'An Antane Kapesh ». Recherches féministes 30.1 (2017). 29-45.

Maracle, Lee. « Oratoire : accéder à la théorie ». Jeannotte, Lamy et St-Amand. 39-44.

Moreton-Robinson, Aileen. «Whiteness, Epistemology and Indigenous Representation». Whitening Race : Essays in Social and Cultural Criticism. Canberra: Aboriginal Studies P, 2004. 75-88.

Pésémapéo Bordeleau, Virginia. L'amant du lac. Montréal : Mémoire d'encrier, 2013.

Sioui, Georges et Dalie Giroux. Histoires de Kanatha : vues et contées : essais et discours, 19912008. Ottawa : PU d'Ottawa, 2008.

St-Amand, Isabelle. «Discours critiques pour l'étude de la littérature autochtone dans l'espace francophone du Québec». Studies in Canadian Literature / Études en littérature canadienne 35.2 (2010). 30-52.

Walton, Hilary. « Un lac comme personnage principal ? Une étude de la volonté et de l'esprit du lac Abitibi dans L'amant du lac de Virginia Pésémapéo Bordeleau ». Voix plurielles 16.1 (2019). 17-29. 


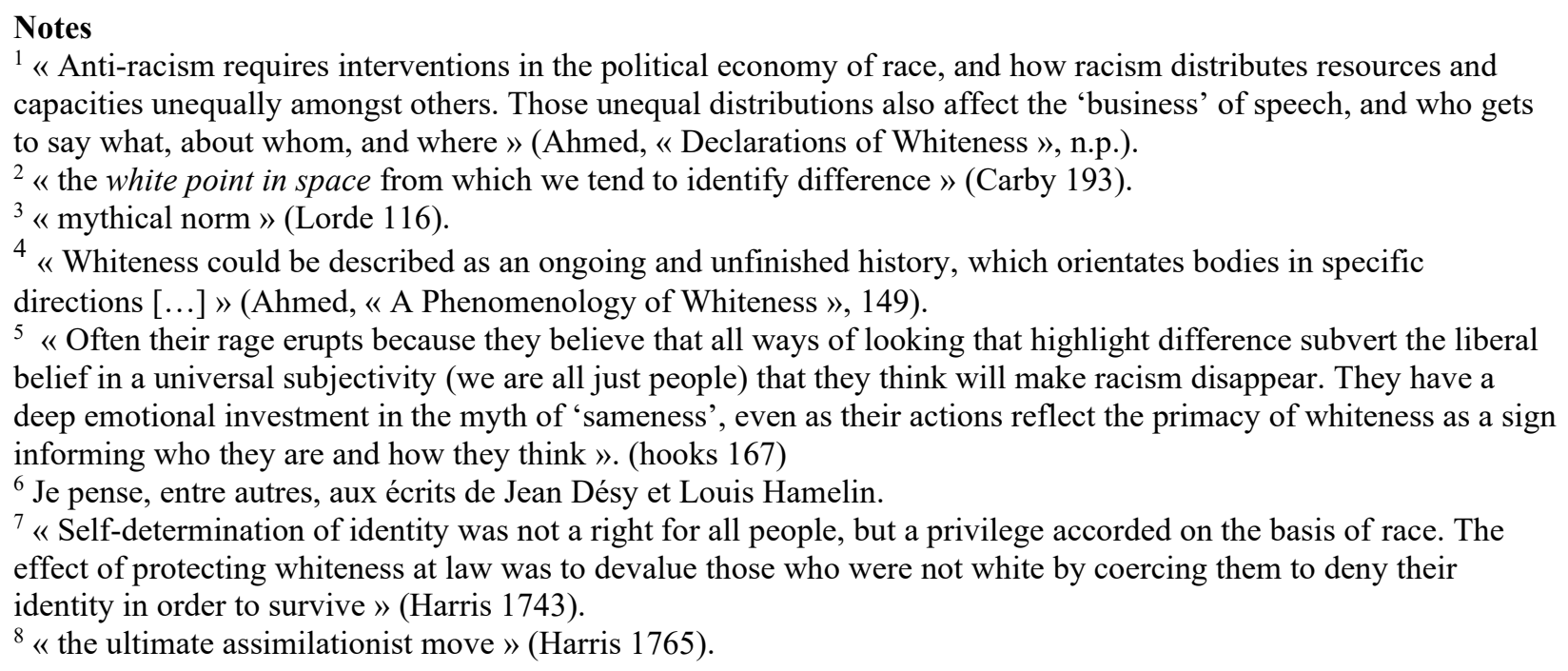

